\title{
Research Article \\ Energy Loss of a Heavy Particle Near 3D Rotating Hairy Black Hole
}

\author{
Jalil Naji $^{1}$ and Hassan Saadat ${ }^{2}$ \\ ${ }^{1}$ Physics Department, Ilam University, P.O. Box 69315-51, Ilam, Iran \\ ${ }^{2}$ Department of Physics, Shiraz Branch, Islamic Azad University, P.O. Box 71555-477, Shiraz, Iran \\ Correspondence should be addressed to Jalil Naji; naji.jalil2020@gmail.com
}

Received 6 November 2013; Accepted 9 January 2014; Published 16 February 2014

Academic Editor: Xiaoxiong Zeng

Copyright (C) 2014 J. Naji and H. Saadat. This is an open access article distributed under the Creative Commons Attribution License, which permits unrestricted use, distribution, and reproduction in any medium, provided the original work is properly cited. The publication of this article was funded by SCOAP $^{3}$.

We consider rotating black hole in 3 dimensions with a scalar charge and discuss energy loss of heavy particle moving near the black hole horizon. We find that drag force was increased by scalar charge while it was decreased due to the rotation of black hole. We also study quasnormal modes.

\section{Introduction}

The lower dimensional theories may be used as toy models to study some fundamental ideas which yield to better understanding of higher dimensional theories, because they are easier to study [1]. Moreover, these are useful for application of AdS/CFT correspondence [2-5]. This paper is indeed an application of AdS/CFT correspondence to probe moving charged particle near the three-dimensional black holes which are recently introduced by $\mathrm{Xu}$ et al. [6, 7] where charged black holes with a scalar hair in $(2+1)$ dimensions and rotating hairy black hole in $(2+1)$ dimensions are constructed, respectively. Here, we are interested in the case of rotating black hole with a scalar hair in $(2+1)$ dimensions. Recently, a charged rotating hairy black hole in 3 dimensions corresponding to infinitesimal black hole parameters was constructed [8]. Also, thermodynamics of such systems is recently studied in $[9,10]$. We consider this background in AdS side as a dual picture of a QCD model as CFT side.

In this paper, we would like to study the motion of a heavy charged particle near the black hole horizon and calculate the energy loss. The energy loss of moving heavy charged particle through a thermal medium is known as the drag force. One can consider a moving heavy particle (such as charm and bottom quarks) near the black hole horizon with the momentum $P$, mass $m$, and constant velocity $v$, which is influenced by an external force $F$. So, one can write the equation of motion as
$\dot{P}=F-\zeta P$, where in the nonrelativistic motion $P=m v$, and in the relativistic motion $P=m v / \sqrt{1-v^{2}}$; also $\zeta$ is called the friction coefficient. In order to obtain drag force, one can consider two special cases. The first case is the constant momentum which yields to obtain $F=(\zeta m) v$ for the norelativistic case. In this case, the drag force coefficient $(\zeta m)$ will be obtained. In the second case, the external force is zero, so one can find $P(t)=P(0) \exp (-\zeta t)$. In other words, by measuring the ratio $\dot{P} / P$ or $\dot{v} / v$, one can determine friction coefficient $\zeta$ without any dependence on mass $m$. These methods lead us to obtain the drag force for a moving heavy particle. The moving heavy particle in context of QCD has dual picture in the string theory in which an open string is attached to the D-brane and stretched to the horizon of the black hole. Therefore, we can apply AdS/CFT correspondence to probe a charged particle (such as a quark) moving through 3D hairy black hole background.

Similar studies are already performed in several backgrounds [11-22]. Most of them considered $\mathcal{N}=2$ and $\mathcal{N}=4$ super Yang-Mills plasma with asymptotically AdS geometries. Also [20] considered 4D Kerr-AdS black holes. All of the mentioned studies used $\mathrm{AdS}_{5} / \mathrm{CFT}_{4}$ correspondence. Now, we are going to consider the same problem in a rotating hairy $3 \mathrm{D}$ background and use $\mathrm{AdS}_{3} / \mathrm{CFT}_{2}$ correspondence [23-25].

This paper is organized as follows. In the next section, we review rotating hairy black hole in $(2+1)$ dimensions. In Section 3, we obtain equation of motion and in Section 4, 
we try to obtain solution and discuss about drag force. In Section 5, we give linear analysis and discuss quasinormal modes. Finally, in Section 6, we summarized our results.

\section{Rotating Hairy Black Hole in $(2+1)$ Dimensions}

Rotating hairy black hole in $(2+1)$ dimensions is described by the following action:

$$
S=\frac{1}{2} \int d^{3} x \sqrt{-g}\left[R-g^{\mu \nu} \nabla_{\mu} \phi \nabla_{\nu} \phi-\frac{R}{8} \phi^{2}-2 V(\phi)\right],
$$

which yields to the following line element [1]:

$$
d s^{2}=-f(r) d t^{2}+\frac{1}{f(r)} d r^{2}+r^{2}(d \psi+\omega(r) d t)^{2}
$$

where

$$
f(r)=3 \beta+\frac{2 \beta B}{r}+\frac{(3 r+2 B)^{2} a^{2}}{r^{4}}+\frac{r^{2}}{l^{2}}
$$

where $a$ is a rotation parameter related to the angular momentum of the solution and $l$ is related to the cosmological constant via $\Lambda=-1 / l^{2} . \beta$ is integration constants depending on the black hole mass:

$$
\beta=-\frac{M}{3}
$$

and scalar charge $B$ is related to the scalar field as

$$
\phi(r)= \pm \sqrt{\frac{8 B}{r+B}}
$$

Also, one can obtain

$$
\begin{gathered}
\omega(r)=-\frac{(3 r+2 B) a}{r^{3}}, \\
V(\phi)=\frac{2}{l^{2}}+\frac{1}{512}\left[\frac{1}{l^{2}}+\frac{\beta}{B^{2}}\right] \phi^{6} .
\end{gathered}
$$

Ricci scalar of this model is given by

$$
R=-\frac{6 r^{6}+36 B l^{2} a^{2} r+30 l^{2} a^{2} B^{2}}{l^{2} r^{6}} .
$$

We can see that Ricci scalar is singular at the origin.

Black hole horizon, which is obtained by $f(r)=0$, may be written as follows:

$$
r_{h}=\frac{4 l}{2 C}\left(1+\sqrt{1-\frac{B C}{3 l^{2}}}\right)
$$

where we defined

$$
C \equiv \frac{2 B M}{27 a^{2}}-\frac{3 l}{B}
$$

\section{The Equations of Motion}

The moving heavy particle near the black hole may be described by the following Nambu-Goto action:

$$
S=-\frac{1}{2 \pi \alpha^{\prime}} \int d \tau d \sigma \sqrt{-G}
$$

where $T_{0}=1 / 2 \pi \alpha^{\prime}$ is the string tension. The coordinates $\tau$ and $\sigma$ are corresponding to the string world-sheet. Also, $G_{a b}$ is the induced metric on the string world-sheet with determinant $G$ obtained as follows:

$$
G=-1-r^{2} f(r)\left(x^{\prime}\right)^{2}+\frac{r^{2}}{f(r)}(\dot{x})^{2},
$$

where we used static gauge in which $\tau=t, \sigma=r$, and the string only extends in one direction $x(r, t)$. Then, the equation of motion is obtained as follows:

$$
\partial_{r}\left(\frac{r^{2} f(r) x^{\prime}}{\sqrt{-G}}\right)-\frac{r^{2}}{f(r)} \partial_{t}\left(\frac{\dot{x}}{\sqrt{-G}}\right)=0 .
$$

We should obtain canonical momentum densities associated with the string as follows:

$$
\begin{aligned}
& \pi_{\psi}^{0}=\frac{1}{2 \pi \alpha^{\prime} \sqrt{-G}} \frac{r^{2}}{f(r)} \dot{x}, \\
& \pi_{r}^{0}=-\frac{1}{2 \pi \alpha^{\prime} \sqrt{-G}} \frac{r^{2}}{f(r)} \dot{x} x^{\prime}, \\
& \pi_{t}^{0}=-\frac{1}{2 \pi \alpha^{\prime} \sqrt{-G}}\left(1+r^{2} f(r)\left(x^{\prime}\right)^{2}\right), \\
& \pi_{\psi}^{1}=\frac{1}{2 \pi \alpha^{\prime} \sqrt{-G}} r^{2} f(r) x^{\prime}, \\
& \pi_{r}^{1}=-\frac{1}{2 \pi \alpha^{\prime} \sqrt{-G}}\left(1-\frac{r^{2}}{f(r)} \dot{x}^{2}\right), \\
& \pi_{t}^{1}=\frac{1}{2 \pi \alpha^{\prime} \sqrt{-G}} r^{2} f(r) \dot{x} x^{\prime} .
\end{aligned}
$$

The simplest solution of the equation of motion is static string described by $x=$ constant with total energy of the form,

$$
E=-\int_{r_{h}}^{r_{m}} \pi_{t}^{0} d r=\frac{1}{2 \pi \alpha^{\prime}}\left(r_{h}-r_{m}\right)=M_{\text {rest }},
$$

where $r_{m}$ is an arbitrary location of D-brane. As we expected, the energy of static particle is interpreted as the remaining mass.

\section{Time Dependent Solution}

In the general case, we can assume that the particle moves with constant speed $\dot{x}=v$; in that case, the equation of motion (12) reduces to

$$
\partial_{r}\left(\frac{r^{2} f(r) x^{\prime}}{\sqrt{-G}}\right)=0,
$$


where

$$
G=-1-r^{2} f(r)\left(x^{\prime}\right)^{2}+\frac{r^{2}}{f(r)} v^{2} .
$$

Equation (15) gives the following expression:

$$
\left(x^{\prime}\right)^{2}=\frac{C^{2}\left(r^{2} v^{2}-f(r)\right)}{r^{2} f(r)^{2}\left(C^{2}-r^{2} f(r)\right)},
$$

where $C$ is an integration constant which will be determined by using reality condition of $\sqrt{-G}$. Therefore, we yield to the following canonical momentum densities:

$$
\begin{aligned}
& \pi_{\psi}^{1}=-\frac{1}{2 \pi \alpha^{\prime}} C, \\
& \pi_{t}^{1}=\frac{1}{2 \pi \alpha^{\prime}} C v .
\end{aligned}
$$

These give us loosing energy and momentum through an endpoint of string:

$$
\begin{aligned}
& \frac{d P}{d t}=\left.\pi_{\psi}^{1}\right|_{r=r_{h}}=-\frac{1}{2 \pi \alpha^{\prime}} C, \\
& \frac{d E}{d t}=\left.\pi_{t}^{1}\right|_{r=r_{h}}=\frac{1}{2 \pi \alpha^{\prime}} C v .
\end{aligned}
$$

As we mentioned before, reality condition of $\sqrt{-G}$ gives us constant $C$. The expression $\sqrt{-G}$ is real for $r=r_{c}>r_{h}$. In the case of small $v$, one can obtain

$$
r_{c}=r_{h}+\left.\frac{r^{2} v^{2}}{f(r)^{\prime}}\right|_{r=r_{h}}+\mathcal{O}\left(v^{4}\right)
$$

which yields to

$$
C=v r_{h}^{2}+\mathcal{O}\left(v^{3}\right)
$$

Therefore, we can write drag force as follows:

$$
\frac{d P}{d t}=-\frac{v r_{h}^{2}}{2 \pi \alpha^{\prime}}+\mathcal{O}\left(v^{3}\right) .
$$

We draw drag force in terms of velocity and in agreement with the previous works such as [11-22]; the value of drag force increased by $v$. In Figure 1, we can see behavior of drag force with rotation parameter and scalar charge. It is shown that the scalar charge increases the value of drag force but the increasing rotational parameter decreases the value of the drag force.

\section{Linear Analysis}

Motion of string yields to small perturbation after late time due to the drag force. In that case, the speed of particle is infinitesimal and one can write $G \approx-1$. Also, we assume that $x=e^{-\mu t}$, where $\mu$ is the friction coefficient. Therefore, one can rewrite the equation of motion as follows:

$$
\frac{f(r)}{r^{2}} \partial_{r}\left(r^{2} f(r) x^{\prime}\right)=\mu^{2} x
$$

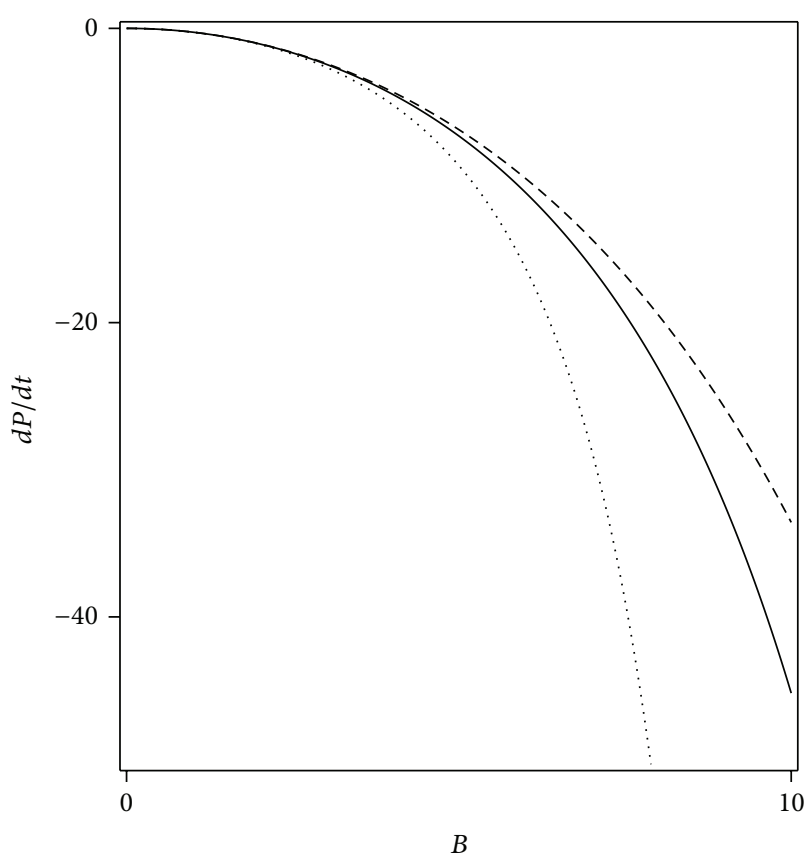

FIGURE 1: Drag force in terms of $B$ for $M=1, l=1$, and $v=0.1$; $a=1.8$ (dotted line), $a=3$ (solid line), and $a=4.2$ (dashed line).

We assume outgoing boundary conditions near the black hole horizon and use the following approximation:

$$
(4 \pi T)^{2}\left(r-r_{h}\right) \partial_{r}\left(r-r_{h}\right) x^{\prime}=\mu^{2} x,
$$

which suggests the following solutions:

$$
x=c\left(r-r_{h}\right)^{-\mu / 4 \pi T},
$$

where $T$ is the black hole temperature. In the case of infinitesimal $\mu$, we can use the following expansion:

$$
x=x_{0}+\mu^{2} x_{1}+\cdots .
$$

Inserting this equation in the relation (24) gives $x_{0}=$ constant, and

$$
x_{1}^{\prime}=\frac{A}{r^{2} f(r)} \int_{r_{h}}^{r_{m}} \frac{r^{2}}{f(r)} d r,
$$

where $A$ is a constant. Assuming near horizon limit enables us to obtain the following solution:

$$
x_{1} \approx \frac{A}{4 \pi T r_{h}^{2}\left(r-r_{h}\right)}\left(-r_{m}+\frac{r_{h}^{2}}{4 \pi T} \ln \left(r-r_{h}\right)\right) .
$$

Comparing (25) and (27) gives the following quasinormal mode condition:

$$
\mu=\frac{r_{h}^{2}}{r_{m}} .
$$

It is interesting to note that these results recover drag force (22) for infinitesimal speed. In Figure 2, we can see behavior of $\mu$ with rotational parameter and scalar charge. We find that scalar charge increases the value of friction coefficient, but the effect of rotation decreases $\mu$. 


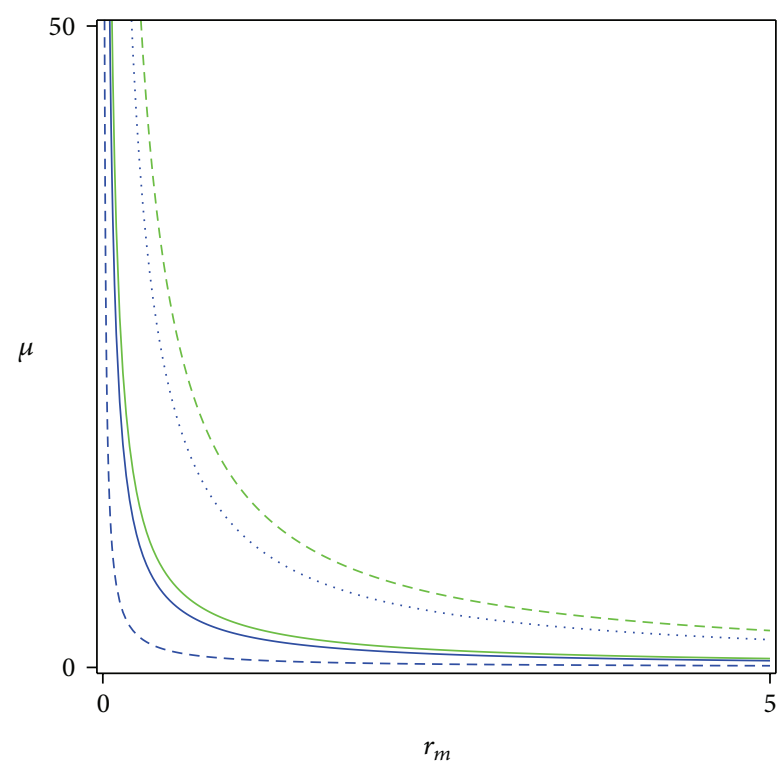

Figure 2: $\mu$ in terms of $r_{m}: B=0.5$ and $a=2$ (blue dashed line), $B=1$ and $a=2$ (blue solid line), $B=2$ and $a=2$ (blue dotted line), and $B=1$ and $a=0.2$ (green dashed line), and $B=1$ and $a=0.4$ (green solid line).

5.1. Low Mass Limit. Low mass limit means that $r_{m} \rightarrow r_{h}$, and we use the following assumptions:

$$
\begin{gathered}
f(r) \approx 4 \pi T\left(r-r_{h}\right), \\
r^{2}=r_{h}^{2}+2 r_{h}\left(r-r_{h}\right)+\cdots,
\end{gathered}
$$

so, by using relation (23) we can write

$$
x(r)=\left(r-r_{h}\right)^{-\mu / 4 \pi T}\left(1+\left(r-r_{h}\right) A+\cdots\right) .
$$

Then, we can obtain constant $A$ as follows:

$$
A=\frac{\mu}{2 \pi T r_{h}-\mu r_{h}} .
$$

It tells that $\mu=2 \pi T$ yields to divergence; therefore we called this a critical behavior of the friction coefficient and found that

$$
\mu_{c}=\frac{3 r_{h}^{6}+B M l^{2} r_{h}^{3}-27 a^{2} l^{2} r_{h}^{2}-54 B a^{2} l^{2} r_{h}-24 B^{2} a^{2} l^{2}}{r_{h}^{5}}
$$

Figure 3 shows behavior of critical friction coefficient with the black hole parameters.

\section{Conclusions}

In this paper, we considered rotating 3D black hole together with a scalar charge as a background where a charged particle moves with speed $v$ and then calculated drag force. We used motivation of AdS/CFT correspondence and string theory method to study motion of charged particle. This is indeed in the context of $\mathrm{AdS}_{3} / \mathrm{CFT}_{2}$ where drag force on moving heavy

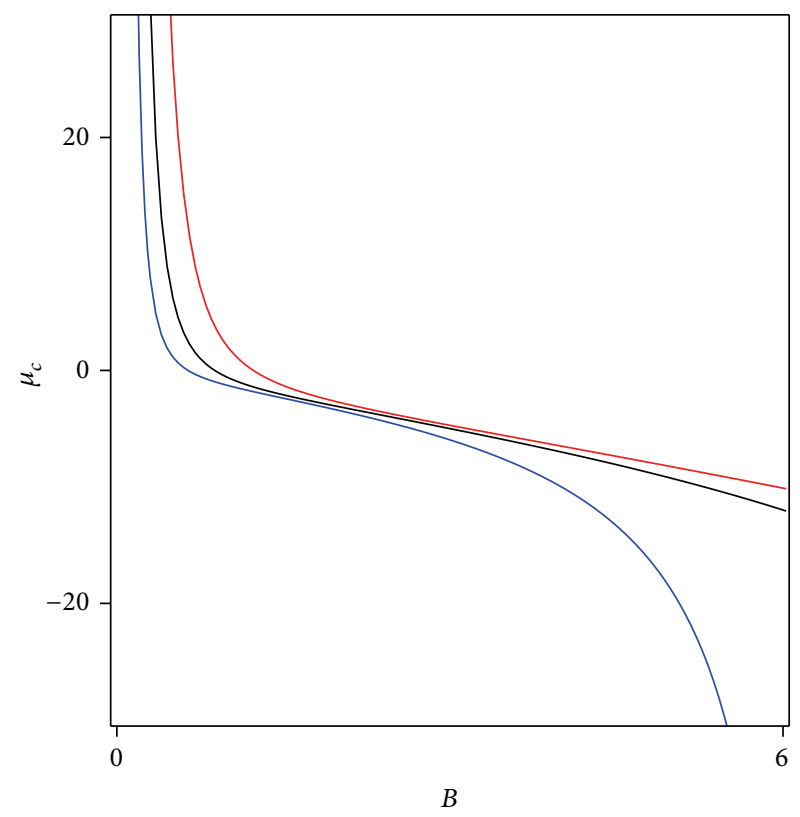

Figure 3: $\mu_{c}$ in terms of $B$ for $M=1$ and $l=1 ; a=1$ (blue line), $a=2$ (black line), and $a=4$ (red line).

particle is calculated. Numerically, we found that the scalar charge increases the value of drag force but rotational parameter decreases the value of the drag force. Therefore, in order to have the most free motion we need to increase $a$ and decrease $B$. It means that $a$ and $B$ may cancel the effect of each other on the drag force. We can find critical values of scalar charge and rotational parameters in which the value of drag force will be infinite as

$$
a_{c}=\sqrt{\frac{2 M}{81 l}} B_{c}
$$

Then, we studied quasinormal modes and obtained friction coefficient $\mu$ which was enhanced by the black hole charge and reduced by rotation. Quasinormal mode analysis also reproduced drag force at slow velocities. It is also possible to study dispersion relations which again reproduce the drag force which was obtained in (22). For the future work, we will consider charged rotating 3D hairy black hole and study drag force.

\section{Conflict of Interests}

The authors declare that there is no conflict of interests regarding the publication of this paper.

\section{References}

[1] M. Hortaçsu, H. T. Özçelik, and B. Yapışkan, "Properties of solutions in $2+1$ dimensions," General Relativity and Gravitation, vol. 35, no. 7, pp. 1209-1221, 2003.

[2] M. Henneaux, C. Martínez, R. Troncoso, and J. Zanelli, "Black holes and asymptotics of $2+1$ gravity coupled to a scalar field," Physical Review D, vol. 65, Article ID 104007, 4 pages, 2002. 
[3] M. Hasanpour, F. Loran, and H. Razaghian, "Gravity/CFT correspondence for three-dimensional Einstein gravity with a conformal scalar field," Nuclear Physics B, vol. 867, no. 2, pp. 483505, 2013.

[4] D. F. Zeng, "An exact hairy black hole solution for AdS/CFT superconductors," In press, http://arxiv.org/abs/0903.2620.

[5] B. Chen, Z. Xue, and J.-j. Zhang, "Note on thermodynamics method of black hole/CFT correspondence," Journal of High Energy Physics, vol. 2013, article 102, 2013.

[6] W. Xu and L. Zhao, "Charged black hole with a scalar hair in (2+1) dimensions," Physical Review D, vol. 87, Article ID 124008, 11 pages, 2013.

[7] L. Zhao, W. Xu, and B. Zhu, "Novel rotating hairy black hole in (2+1)-dimensions," In press, http://arxiv.org/abs/1305.6001.

[8] J. Sadeghi, B. Pourhassan, and H. Farahani, "Rotating charged hairy black hole in $(2+1)$ dimensions and particle acceleration," In press, http://arxiv.org/abs/1310.7142.

[9] A. Belhaj, M. Chabab, H. EL Moumni, and M. B. Sedra Critical behaviors of 3D black holes with a scalar hair," In press, http:// arxiv.org/abs/1306.2518.

[10] J. Sadeghi and H. Farahani, "Thermodynamics of a charged hairy black hole in (2+1) dimensions," In press, http://arxiv.org/ abs/1308.1054.

[11] C. Hoyos-Badajoz, "Drag and jet quenching of heavy quarks in a strongly coupled Script $\mathcal{N}=2^{*}$ plasma," Journal of High Energy Physics, vol. 2009, no. 9, article 068, 2009.

[12] J. Sadeghi and B. Pourhassan, "Drag force of moving quark at $\mathcal{N}=2$ supergravity," Journal of High Energy Physics A, vol. 2008, no. 12, article 026, 2008.

[13] C. P. Herzog, A. Karch, P. Kovtun, C. Kozcaz, and L. G. Yaffe, "Energy loss of a heavy quark moving through $\mathcal{N}=4$ supersymmetric Yang-Mills plasma," Journal of High Energy Physics A, vol. 2006, no. 7, article 013, 2006.

[14] J. Sadeghi, B. Pourhassan, and S. Heshmatian, "Application of AdS/CFT in quark-gluon plasma," Advances in High Energy Physics, vol. 2013, Article ID 759804, 13 pages, 2013.

[15] C. P. Herzog, "Energy loss of a heavy quark from asymptotically AdS geometries," Journal of High Energy Physics A, vol. 2006, no. 9, article 032, 2006.

[16] S. S. Gubser, "Drag force in AdS/CFT," Physical Review D, vol. 74, no. 12, Article ID 126005, 4 pages, 2006.

[17] E. Nakano, S. Teraguchi, and W. Y. Wen, "Drag force, jet quenching, and AdS/QCD," Physical Review D, vol. 75, no. 8, Article ID 085016, 6 pages, 2007.

[18] E. Cáceres and A. Güijosa, "Drag force in a charged $\mathcal{N}=4$ SYM plasma," Journal of High Energy Physics A, vol. 2006, no. 11, article 077, 2006.

[19] J. F. Vazquez-Poritz, "Drag force at finite't Hooft coupling from AdS/CFT, In press, http://arxiv.org/abs/0803.2890.

[20] A. N. Atmaja and K. Schalm, "Anisotropic drag force from 4D Kerr-AdS black holes," http://arxiv.org/abs/1012.3800.

[21] B. Pourhassan and J. Sadeghi, "STU-QCD correspondence," Canadian Journal of Physics, vol. 91, no. 12, pp. 995-1019, 2013.

[22] E. Caceres and A. Guijosa, "On drag forces and jet quenching in strongly coupled plasmas," Journal of High Energy Physics, vol. 2006, no. 12, article 068, 2006.

[23] P. Kraus, "Lectures on black holes and the $A d S_{3} / C F T_{2}$ correspondence," in Supersymmetric Mechanics. Vol. 3, vol. 755 of Lecture Notes in Physics, pp. 193-247, Springer, Berlin, Germany, 2008.
[24] R. Borsato, O. Ohlsson Sax, and A. Sfondrini, "All-loop Bethe ansatz equations for $A d S_{3} / C F T_{2}$," Journal of High Energy Physics, vol. 4, article 116, 2013.

[25] D. Momeni, M. Raza, M. R. Setare, and R. Myrzakulov, "Analytical holographic superconductor with backreaction using $\mathrm{AdS}_{3} / \mathrm{CFT}_{2}$," International Journal of Theoretical Physics, vol. 52, no. 8, pp. 2773-2783, 2013. 

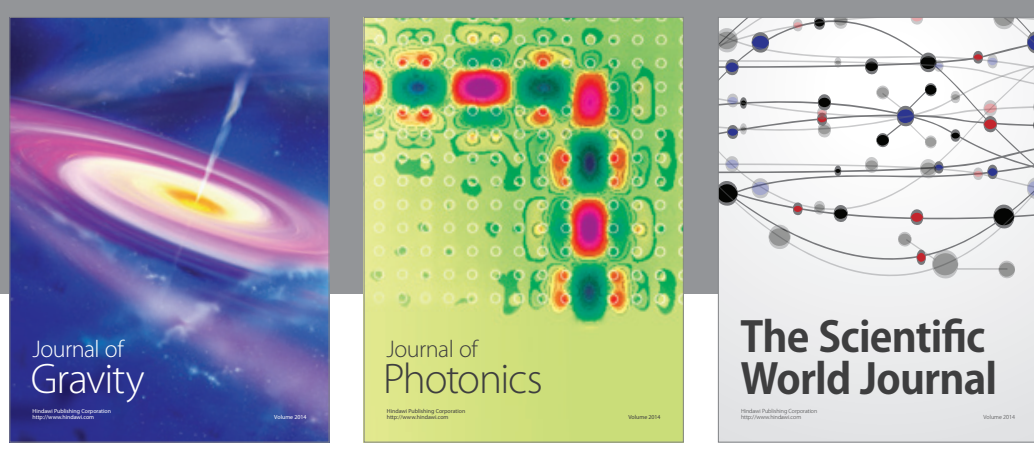

The Scientific World Journal
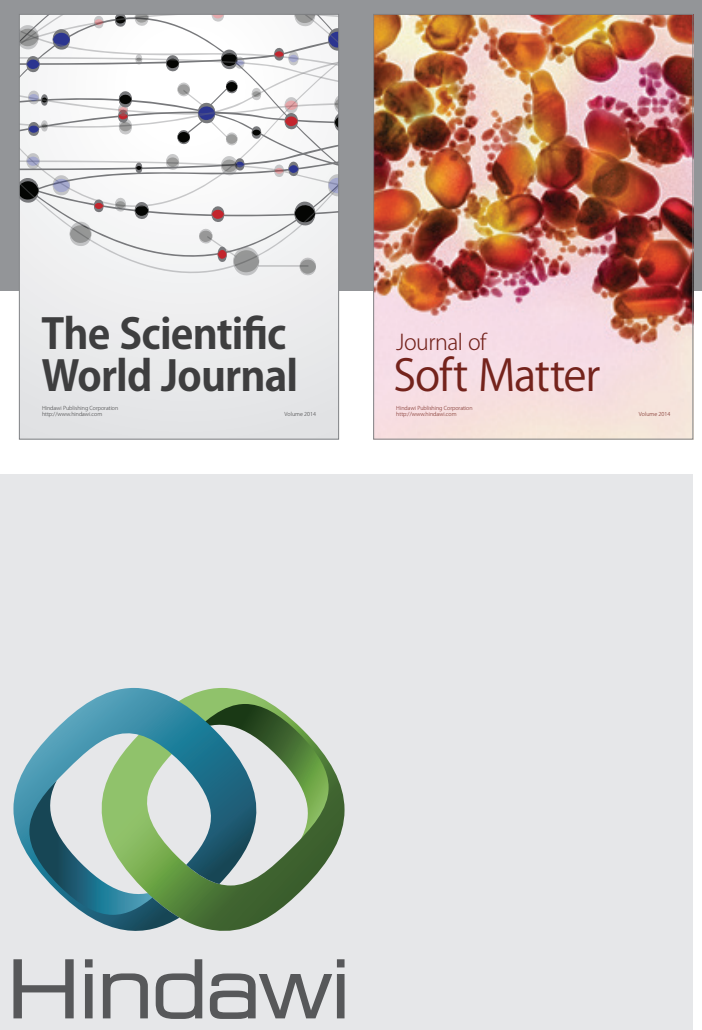

Submit your manuscripts at

http://www.hindawi.com

nternational Journal of

Statistical Mechanics
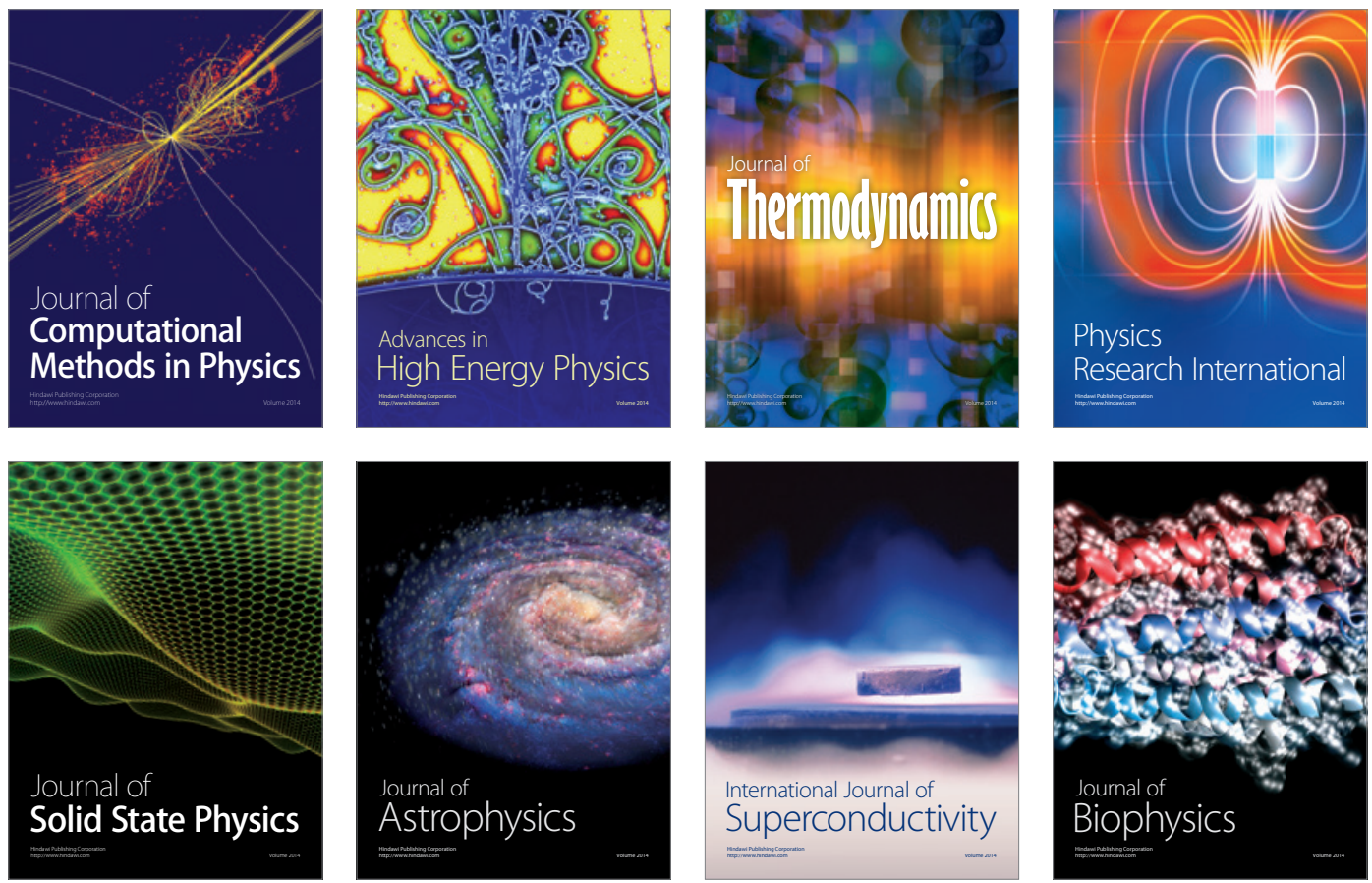
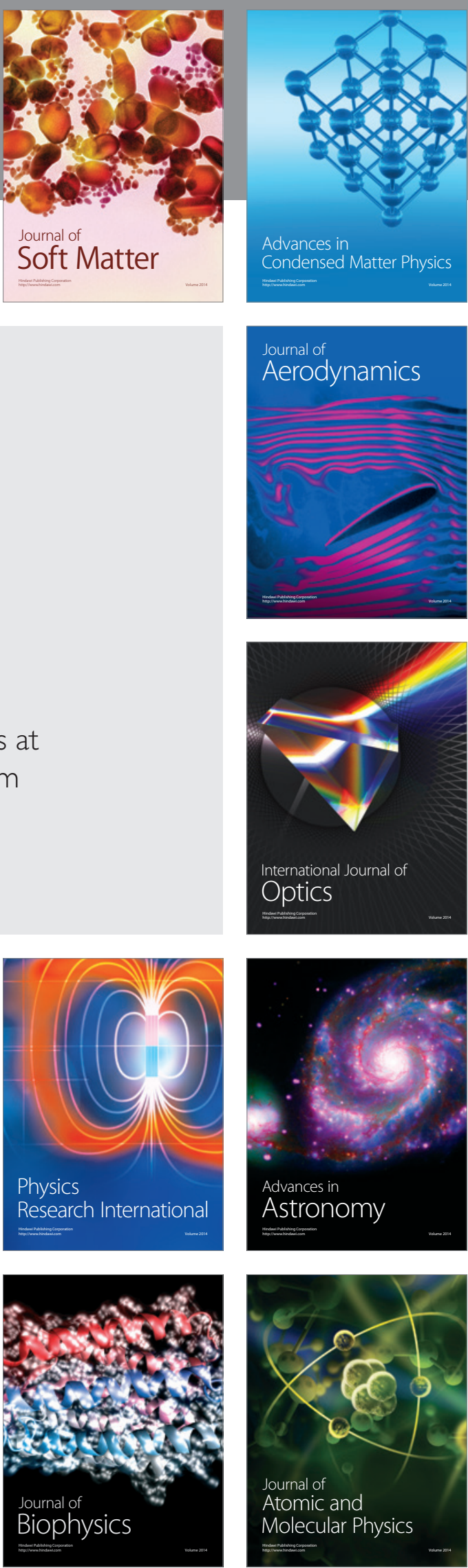